$r$ e vista docência do ensino

\title{
SEÇÃO: ARTIGOS
}

\section{Exame Clínico Objetivo Estruturado na avaliação formativa: percepção de estudantes de Medicina}

\author{
Nilson Chaves Junior ${ }^{1}$, Maria Cristina Almeida de Souza ${ }^{2}$, \\ João Carlos de Souza Côrtes Junior ${ }^{3}$, Paula Pitta de Resende Côrtes ${ }^{4}$, \\ Milton de Arruda Martins ${ }^{5}$
}

\section{RESUMO}

A formação em medicina demanda o desenvolvimento de competências pelos estudantes, exigindoIhes mobilização de conhecimentos, habilidades e atitudes para lidarem com situações da prática profissional. O Exame Clínico Objetivo Estruturado (OSCE) constitui-se em estratégia para avaliar competências em ambientes simulados. Este estudo, uma pesquisa quantitativa, descritiva e transversal, teve por objetivo avaliar a percepção dos estudantes do sétimo e do oitavo períodos de um curso de Medicina sobre alguns fatores que interferiram no seu desempenho durante o OSCE. A amostra, não probabilística por conveniência, foi constituída por 101 estudantes, representando $64,7 \%$ do universo. A coleta de dados foi realizada virtualmente através de um formulário Google Forms, enviado aos estudantes por meio de seus endereços eletrônicos. Concluiu-se que a percepção dos estudantes foi positiva em relação ao exame, ainda que ajustes se revelem necessários, sinalizando para a viabilidade e factibilidade de novas edições do OSCE no curso de Medicina.

Palavras-chave: Avaliação educacional. Competência clínica. Educação médica. Educação baseada em competências. Exame Clínico Objetivo Estruturado.

\section{Como citar este documento - ABNT}

CHAVES JUNIOR, Nilson et al. Exame Clínico Objetivo Estruturado na avaliação formativa: percepção de estudantes de Medicina. Revista Docência do Ensino

Recebido em: 24/08/2020 Superior, Belo Horizonte, v. 11, e024884, p. 1-16, 2021. DOI: https://doi.org/10.35699/2237-5864.2021.24884.

\footnotetext{
${ }^{1}$ Universidade de Vassouras (UV), Vassouras, RJ, Brasil. ORCID ID: http://orcid.org/0000-0003-3312-6649. E mail: nchavesjr@me.com ${ }^{2}$ Universidade de Vassouras (UV), Vassouras, RJ, Brasil. ORCID ID: https://orcid.org/0000-0001-7631-723X. E mail: mcas.souza@uol.com.br ${ }^{3}$ Universidade de Vassouras (UV), Vassouras, RJ, Brasil. ORCID ID: http://orcid.org/0000-0002-3263-5129. E mail: joaocortes@yahoo.com.br ${ }^{4}$ Universidade de Vassouras (UV), Vassouras, RJ, Brasil. ORCID ID: http://orcid.org/0000-0001-5935-7045. E mail: paulapitta@yahoo.com.br ${ }^{5}$ Universidade de São Paulo (USP), São Paulo, SP, Brasil. ORCID ID: https://orcid.org/0000-0001-9690-9371. E mail: mmartins@usp.br
} 


\section{Examen Clínico Objetivo Estructurado en evaluación formativa: percepción de los estudiantes de Medicina}

\section{RESUMEN}

La formación en medicina requiere el desarrollo de habilidades por parte de los estudiantes, requiriendo la movilización de conocimientos, habilidades y actitudes para afrontar situaciones de práctica profesional. El Examen Clínico Objetivo Estructurado (OSCE) es una estrategia para evaluar competencias en entornos simulados. Este estudio, cuantitativo, descriptivo y transversal, tuvo como objetivo evaluar la percepción de los estudiantes del séptimo y octavo ciclo de una carrera de Medicina sobre algunos factores que pueden interferir en su desempeño durante el OSCE. La muestra, no probabilística por conveniencia, estuvo formada por 101 estudiantes, lo que representa el 64,7\% del universo. La recolección de datos se realizó virtualmente a través de un formulario de Google, enviado a los estudiantes a través de sus direcciones de correo electrónico. Se concluyó que la percepción de los estudiantes fue positiva en relación al examen, aunque son necesarios ajustes, lo que indica la viabilidad y factibilidad de nuevas ediciones del OSCE en la carrera de Medicina.

Palabras clave: Evaluación educacional. Competencia clínica. Educación médica. Educación basada en competencias. Examen Clínico Objetivo Estructurado.

\section{Objective Structured Clinicall Examination in formaltive assessment: perception of Medicine students}

\section{ABSTRACT}

Training in medicine requires development of skills by students, such as knowledge mobilization, skills, and attitudes to deal with situations on professional practice. The Objective Structured Clinical Examination (OSCE) is a strategy to assess competencies in simulated environments. This study, a quantitative, descriptive and cross-sectional one, aimed to assess the perception of students in the seventh and eighth period of a Medical program on some factors that may interfere with their performance during the OSCE. The sample, non-probabilistic for convenience, consisted of 101 students, representing $64.7 \%$ of the universe. Data collection was performed virtually through a Google Form, sent to students through their email addresses. It was concluded that the students' perception about the exam was positive, although adjustments are necessary, drawing attention to the feasibility of new editions of the OSCE in the Medical program.

Keywords: Educational measurement. Clinical competence. Medical education. Competency-based education. Objective Structured Clinical Examination. 


\section{INTRODUÇÃO}

As Diretrizes Curriculares Nacionais para os cursos de graduação em Medicina preconizam uma formação baseada no desenvolvimento de competências (BRASIL, 2014). O ensino por competências exige que o estudante mobilize os conhecimentos, as habilidades e as atitudes para lidar com situações, problemas e dilemas da práxis (PERRENOUD, 1999). Competência, para fins de organização de currículos na área de saúde, deve ser concebida como a capacidade de mobilizar, articular e colocar em prática conhecimentos, habilidades e atitudes necessárias ao desempenho efetivo das atividades requeridas no contexto do trabalho.

Nos últimos anos, tem ganhado destaque a avaliação de competências clínicas na área da saúde (CHAVES et al., 2019). Especificamente no ensino médico, a avaliação do desempenho clínico torna-se imprescindível, haja vista que, já na graduação, os estudantes realizam atendimentos e procedimentos cuja resolutividade está diretamente relacionada à construção destas competências. Nesse contexto, diversos métodos de avaliação de competências clínicas têm sido propostos, com destaque para o Objective Structured Clinical Examination (OSCE), apresentado por Harden e Gleeson, na década de setenta (NEVES et al., 2016; SILVA et al., 2019; TIBÉRIO et al., 2012).

O OSCE, traduzido para a língua portuguesa como Exame Clínico Objetivo Estruturado, constitui-se em uma estratégia que viabiliza avaliar habilidades clínicas e atitudes em ambientes simulados, seja por meio da interação dos estudantes com fictícios pacientes ou pelo uso de recursos didáticos (MEGALE; GONTIJO; MOTA, 2012). Sua operacionalização exige a montagem de estações, em que cada uma reproduz um ambiente na qual determinada habilidade pode ser observada pelo avaliador de acordo com um roteiro prédefinido de observação - um checklist -, onde estão listadas as ações cuja realização se espera pelos estudantes. Nesta modalidade avaliativa, o estudante tanto aplica o processo de raciocínio e de tomada de decisão quanto faz uso das habilidades motoras para execução de procedimentos, além de exercitar suas atitudes para atender o paciente simulado, configurando uma avaliação por competências (BUSTAMANTE et al., 2000; CHAVES et al., 2019; MEGALE; GONTIJO; MOTA, 2012). As competências a serem demonstradas podem estar inter-relacionadas nas diversas estações ou, diferentemente, podem ser independentes, inexistindo uma interface direta (NEVES et al., 2016).

Um outro método de avaliação prática de habilidades clínicas é o Mini-CEX (Mini Clinical Evaluation Exercise), ou mini-exercício clínico, que exige, no entanto, a presença de pacientes, distinguindo-se do OSCE, que é realizado em ambientes simulados. É um instrumento de observação direta de desempenho, que permite ao professor avaliar o estudante enquanto este realiza uma consulta objetiva e rápida, focada em determinada 
necessidade do paciente. Sua principal característica é reproduzir, da maneira mais fiel possível, a rotina do profissional em seu local de trabalho (ABBADE, 2012).

Como vantagens apresentadas pelo OSCE, destacam-se a validade, a viabilidade e a confiabilidade, pois, distintamente das avaliações executadas em espaços reais, essa permite a padronização dos cenários e do exame, viabilizando, assim, que todos os estudantes sejam avaliados sob as mesmas condições (NEVES et al., 2016; TIBÉRIO et al., 2012). Adicionalmente, o OSCE contempla uma etapa de autoavaliação, representada por um momento de devolutiva dos resultados (feedback) aos estudantes e de interação com o professor, instrumentos válidos para incrementar a percepção dos aprendizes sobre seu desempenho, possibilitando que exerçam a criticidade em relação à sua formação (CHAVES et al., 2019).

O momento do feedback é uma oportunidade ímpar para identificar fortalezas e fragilidades no processo formativo, assim como para elaborar propostas para o seu aprimoramento. Professor e estudante reconhecem, a partir de uma interação dialógica, o que precisa ser otimizado na aprendizagem, viabilizando o planejamento de estratégias de correção (GONTIJO; ALVIM; REIS, 2018). O feedback realizado com os estudantes é considerado um indicador de eficácia e de confiança durante o processo avaliativo (FERREIRA et al., 2020; FRANCO et al., 2015; KILLINGLEY; DYSON, 2016).

Escolas médicas de mais de 50 países já utilizam o OSCE em seus processos avaliativos, com bons resultados (EBERHARD et al., 2011; SILVA et al., 2019), sendo esse exame considerado "padrão ouro" para avaliar objetivamente as competências médicas (GUPTA; DEWAN; SINGH, 2010), uma vez que não se restringe à avaliação da dimensão cognitiva, mas inclui também a capacidade de colocar em prática o conhecimento construído (BUSTAMANTE et al., 2000). Como desvantagem, o OSCE demanda grande logística e força de trabalho, sendo necessário planejamento prévio, número suficiente de avaliadores, estações de acordo com o número de estudantes avaliados, espaço físico para acomodar aqueles que aguardam o momento da avaliação, impossibilidade de simular procedimentos invasivos em pacientes reais, ansiedade dos estudantes, assim como apresenta um custo mais elevado em comparação ao investido na realização de outros métodos de avaliação (CHAVES et al., 2019; NEVES et al., 2016; WILBY; DIAB, 2016; ZARTMAN et al., 2002).

Tendo em vista a proposta dos gestores acadêmicos do curso de graduação em Medicina da Universidade de Vassouras em sistematizar, a partir de 2021, a realização do OSCE nos processos avaliativos das disciplinas, inserindo-o como um dos métodos avaliativos nas disciplinas do sexto ao oitavo período da matriz curricular, torna-se fundamental analisar a percepção do estudante sobre esse exame. Assim, o objetivo desta pesquisa foi avaliar a percepção dos estudantes do sétimo e do oitavo períodos do curso sobre alguns fatores que 
podem interferir no seu desempenho durante a realização do OSCE, de modo que os resultados da pesquisa possam subsidiar ações para o aperfeiçoamento da realização do exame.

\section{MATERIAIS E MÉTODOS}

Trata-se de uma pesquisa quantitativa, descritiva e transversal, cujos dados foram coletados por meio de um instrumento estruturado (adaptado de SILVA et al., 2019), contendo dez questões objetivas (QUADRO 1). A amostra, não probabilística por conveniência, foi constituída por estudantes do sétimo e do oitavo períodos do curso de Medicina da Universidade de Vassouras, que participaram da edição do OSCE no curso, realizada em 2018. Os critérios de inclusão foram o estudante estar matriculado nesses períodos em 2020 e ter participado da edição do OSCE promovido pela disciplina "Fundamentos da Cirurgia", em 2018.

Esclarece-se que, tendo em vista o isolamento social determinado por órgãos governamentais durante a pandemia Covid-19, a coleta de dados foi realizada virtualmente por meio de formulário Google Forms, enviado nos meses de abril e maio de 2020 aos estudantes por meio de seus endereços eletrônicos. O participante foi informado que a devolução do instrumento, devidamente preenchido, implicava em seu consentimento livre e esclarecido de participação da pesquisa, tendo ciência dos seus riscos e benefícios.

A presente pesquisa foi aprovada pelo Comitê de Ética da Universidade de Vassouras, mediante Parecer $n^{\circ} .3 .908 .676$, de 10/03/2020 (UNIVERSIDADE DE VASSOURAS, 2020). Em sua realização, obedeceu-se a todos os princípios éticos da Declaração de Helsinque (ASSOCIAÇÃO MÉDICA MUNDIAL, 1964), bem como aos preceitos e orientações referentes à Ética em Pesquisa envolvendo Seres Humanos, conforme dispositivos presentes na Resolução n 466 de 12 de dezembro de 2012 (BRASIL, 2012). 
Período em que está matriculado: Gênero:

Prezado participante, após ler as perguntas, marque a sua opção sobre o Exame Clínico Objetivo Estruturado (OSCE):

1- As tarefas solicitadas foram demonstradas durante as aulas práticas? ( ) Sim ( ) Não

2- Os temas nas estações foram relevantes para minha formação em Medicina? ( ) Sim ( ) Não

3- O tempo para cada estação foi adequado? ( ) Sim ( ) Não

4- As orientações fornecidas nas estações foram adequadas? ( ) Sim ( ) Não

5- O OSCE ajudou-te a identificar os pontos fortes e os fracos em habilidades clínicas? ( ) Sim ( ) Não

6- O feedback foi útil e ofereceu oportunidade de aprendizagem? ( ) Sim ( ) Não

7- Suas habilidades foram avaliadas de forma justa? ( ) Sim ( ) Não

8- O OSCE me estimulou a buscar mais informações sobre os temas? ( ) Sim ( ) Não

9- O comportamento dos avaliadores o incomodou? ( ) Sim ( ) Não

10- Sentiu-se estressado ao realizar a prova? ( ) Sim ( ) Não

Quadro 1 - Instrumento para coleta de dados

Fonte: adaptado de SILVA et al., 2019.

O circuito, montado no laboratório de habilidades e simulação, foi constituído por quatro estações, compondo uma série com número inferior à média citada em outros estudos, de dez a vinte estações (CHAVES JUNIOR, 2018), mas cujo quantitativo atendia aos objetivos da disciplina. Para cada uma das estações, foi disponibilizado ao estudante o tempo de oito minutos. Os temas das estações, que estavam numeradas de um a quatro, foram respectivamente: noções de prevenção de infecções para alas afins do centro cirúrgico (retirada de adornos, colocação de gorro, de máscara, de proteção dos pés e escovação das mãos); colocação de avental cirúrgico e de luvas estéreis; indicação e técnica para realização de cateterismo nasogástrico; identificação e terapêutica adequada do manejo de uma lesão de pele.

Antes da aplicação do método, os estudantes permaneceram em uma sala onde receberam informações sobre o objetivo e as normas do exame. No acesso externo à cada estação, foi afixada a descrição de todos os itens que seriam avaliados, de modo que o estudante conhecesse previamente todas as ações que deveria realizar antes da sua entrada na estação do OSCE.

Um checklist padronizado permitiu que o avaliador observasse e anotasse todas as ações e condutas realizadas por cada estudante. Para confecção dos escores de cada estudante, adotou-se um critério de nota que contemplou itens distintos em cada estação, de modo que foram avaliados aspectos cognitivos (conhecimentos), psicomotores (habilidades clínicas) e afetivos (atitudes). Estabeleceram-se, para cada estação, pontuações diferentes de acordo com o item, com o seguinte critério: acerto para todos os itens avaliados, 1,0 
ponto; erro de apenas um item, 0,75 pontos; acerto da metade dos itens, 0,5 pontos; acerto de apenas um item, 0,25 pontos; e sem acerto de itens, nenhum ponto. A soma dos pontos de todos os itens do checklist em cada estação constituiu a pontuação final.

Ao término de cada estação, foi dado o feedback ao estudante sobre seu desempenho, por meio da leitura dos escores obtidos e a sinalização das competências que não foram suficientemente demonstradas no OSCE, contribuindo para a aprendizagem autodirecionada e para a reflexão crítica, elementos imprescindíveis à aprendizagem significativa (SAMPAIO; PRICINOTE; PEREIRA, 2014). Os professores atentaram-se para que a informação sobre o desempenho fosse capaz de promover um desenvolvimento positivo do estudante.

\section{RESULTADOS}

Dos 156 estudantes matriculados nos períodos selecionados para a pesquisa, 101 participaram da pesquisa, representando $64,7 \%$ do universo. Declararam ser do gênero feminino $73 \%$ dos participantes.

As tarefas solicitadas nas estações do OSCE foram previamente demonstradas durante as aulas práticas, na percepção de $89,1 \%$ dos estudantes, e, para $98 \%$ deles, os temas abordados foram relevantes para a formação em Medicina. Contudo, para $27,7 \%$ dos estudantes, o tempo de oito minutos destinado à atividade em cada estação foi insuficiente. As orientações fornecidas nas estações foram adequadas na opinião de $91,1 \%$ dos discentes.

A contribuição do OSCE para a identificação dos pontos fortes e dos pontos fracos nas habilidades clínicas dos estudantes foi positivamente informada por $79,2 \%$ da amostra, e $81,1 \%$ dos estudantes apontaram que o feedback foi útil e ofereceu-Ihes uma oportunidade de aprendizagem. Para 77,2\% dos participantes, as habilidades foram avaliadas de forma justa, e o OSCE estimulou $67,3 \%$ dos aprendizes a buscarem mais informações sobre os temas.

Em relação à estabilidade emocional durante a avaliação das competências, apenas $22,8 \%$ dos estudantes se sentiram incomodados com o comportamento dos avaliadores, enquanto $51,5 \%$ consideraram que a avaliação Ihes causou estresse durante sua realização.

\section{DISCUSSÃO}

A Universidade de Vassouras, no sul do estado do Rio de Janeiro, oferta, há 51 anos, o curso de graduação em Medicina, cujo currículo contempla diversos processos avaliativos, que, dotados de caráter formativo, contribuem para a graduação de médicos éticos, resolutivos e 
com perfil preconizado pelas Diretrizes Curriculares Nacionais para Cursos de Graduação em Medicina (BRASIL, 2014).

Para graduar egressos com esse perfil, o projeto pedagógico do curso orienta para a realização de uma avaliação contextualizada, válida e confiável com o objetivo de verificar a construção de conhecimento e o desenvolvimento de habilidades e atitudes pelo estudante. Entre as avaliações realizadas, destaca-se o OSCE, cuja implementação vem sendo gradativa (UNIVERSIDADE DE VASSOURAS, 2019), tendo em vista a pouca familiarização da comunidade acadêmica com este método avaliativo. A inovação foi iniciada na disciplina "Fundamentos da Cirurgia", pois foi aquela cuja equipe docente aceitou, de imediato, o desafio de operacionalizar o OSCE, além de ser composta por professores formadores de opinião, com resiliência suficiente para receber eventuais críticas dos pares e também para identificar a necessidade de adequações às futuras edições do exame, que poderão ser potencializadas a partir dos resultados dessa pesquisa.

Após análise crítica da experiência pela equipe docente executora, tendo em vista o protagonismo discente no processo ensino-aprendizagem e a premissa de que o processo avaliativo exige constantes reflexões e revisões de seus instrumentos, o Núcleo Docente Estruturante (NDE) e o colegiado do curso decidiram que era necessário verificar a percepção dos estudantes sobre o OSCE, ratificando o pressuposto de que a análise da qualidade de qualquer avaliação é essencial para que ajustes sejam efetivados (FRANCO et al., 2015).

Coletados os dados sobre a percepção dos estudantes em relação ao OSCE, a análise das respostas dos participantes permitiu verificar que adequações serão, de fato, necessárias às edições futuras do exame. Os resultados viabilizaram constatar a aplicabilidade prática das estações do OSCE, pois $98 \%$ da amostra opinou que os temas das estações foram relevantes para a sua formação profissional, evidenciando que a elaboração de ambientes simulados deve ser a mais fidedigna possível da realidade, simulando casos/situações da prática médica, conferindo aos cenários maior relevância e significado (FRANCO et al., 2015; SILVA et al., 2019; ZARTMAN et al., 2002). Resultado semelhante foi encontrado em investigação sueca (87\%), sugerindo que uma das premissas do OSCE foi respeitada, a de abordar os aspectos relevantes ao exercício profissional (SILVA et al., 2019; ZARTMAN et al., 2002). Diferentemente dos resultados encontrados na pesquisa desenvolvida na Universidade de Vassouras, Siddiqui (2013), ao analisar a percepção de aprendizes sobre a aplicação do OSCE, observou que a maior parte dos estudantes sinalizou que os conteúdos demandados na avaliação não foram de grande relevância para a futura prática profissional.

Em relação ao tempo destinado à realização da atividade exigida em cada estação, existe um percentual de estudantes para os quais há necessidade de ampliação dos minutos 
destinados às estações, assemelhando-se aos achados de outros pesquisadores (SILVA et al., 2019; ZARTMAN et al., 2002), embora o tempo para cada estação, de oito minutos, atende ao citado em estudo australiano que preconiza de cinco a oito minutos por estação (NULTY et al., 2011). O tempo destinado às estações foi considerado também insuficiente por estudantes da Etiópia ao externarem suas percepções em relação ao OSCE (ATARO; BESSA; ASAMINEW, 2020).

Baseando-se no fato de que estudantes aprendem em ritmos distintos e de que o OSCE é uma avaliação critério referenciada sem objetivo de selecionar, mas sim de ensinar (SOUZA, 2012), essa é uma informação que deve ser considerada pelos gestores da instituição em futuras edições do exame, atendendo ao preconizado por Idris et al. (2014) de que a realização demanda um tempo adequado para realização das tarefas pelos estudantes. Nesse sentido, Smee (2003) aponta que o tempo estabelecido para as estações constitui uma das limitações apresentadas pelo OSCE, pois isso pode desconstruir a relação médicopaciente e prejudicar a avaliação formativa pretendida. Entende-se por avaliação formativa aquela que, além de comprometida com o fomento ao aprendizado significativo, tem, entre seus atributos, a validade e o impacto educacional.

Constata-se que as informações e orientações fornecidas nas estações foram adequadas, de acordo com o sinalizado por $91,1 \%$ dos estudantes, o que demonstra que essa etapa, essencial ao desempenho satisfatório do estudante, foi exitosa no OSCE realizado na disciplina de "Fundamentos de Cirurgia".

A contribuição do OSCE para identificação dos pontos fortes e dos pontos fracos em relação às habilidades clínicas pelos estudantes foi positivamente informada por $79,2 \%$ dos participantes e os ajudará a enfrentar situações clínicas reais, evidenciando o alcance dos objetivos do exame. Zimmermann (2019) cita que a realização do OSCE aumentou a segurança e autoconfiança dos estudantes, possibilitando reafirmarem o que sabiam assim como o que ainda precisavam saber com vistas a melhorar seu desempenho.

Ao avaliarem a percepção de alunos de Medicina sobre o OSCE, Nasir et al. (2014) constataram que os discentes também consideraram que o exame lhes possibilitou um diagnóstico a respeito do seu processo de construção do conhecimento. Em decorrência disso, os estudantes relataram que isso favoreceu o posterior planejamento do estudo, permitindo-lhes investir mais tempo nos conteúdos e aspectos relacionados às deficiências na formação médica, identificadas durante a realização do exame (SILVA et al., 2019).

O feedback, importante instrumento de ensino e aprendizagem, foi classificado como útil para um expressivo número de estudantes participantes dessa pesquisa, corroborando os achados que evidenciam a importância da autoavaliação e da discussão do conteúdo 
avaliado com professores e colegas, ratificando a relevância da aprendizagem colaborativa (CHAVES et al., 2019; FRANCO et al., 2015; SILVA et al., 2019; ZARTMAN et al., 2002). Também registram-se estudos desenvolvidos por Silva et al. (2019), em que foi verificado um expressivo percentual de estudantes (62,2\%) que concordou que suas habilidades foram avaliadas de forma justa durante a prova. Na pesquisa realizada no curso de Medicina da Universidade de Vassouras, observou-se que há um percentual de alunos $(22,8 \%)$ que julga ser injusta a forma como suas habilidades foram avaliadas, revelando a necessidade de identificar as possíveis causas dessa percepção a fim de eliminar iniquidades na realização da avaliação.

Dos participantes dessa pesquisa, $67,3 \%$ informaram que o OSCE os estimulou a buscar mais informações sobre os temas contemplados no exame, que representa percentual inferior aos registrados por Silva et al. (2019). Tendo em vista que um dos objetivos do curso é fomentar a autonomia intelectual dos futuros médicos, há necessidade de se pensar em estratégias capazes de otimizar a motivação estudantil para a curiosidade, para o estudo individual e para o interesse na pesquisa por informações imprescindíveis à formação na área da saúde.

O incômodo gerado pelo comportamento dos avaliadores, citado por $22,8 \%$ dos estudantes, é um dado significativo, mas há também registro desse fato em OSCE realizado por outras instituições (SILVA et al., 2019), sinalizando que medidas necessitam ser implementadas a fim de que esse fator não interfira no desempenho dos estudantes durante exames vindouros, evitando desvios nos objetivos da avaliação.

Quando se trata do comportamento dos examinadores, Gupta, Dewan e Singh (2010) citam que somente a presença desse indivíduo, mesmo que esse não diga nada durante o exame, pode ser prejudicial ao desempenho do estudante, devido à linguagem corporal transmitida, que tende a ser observada pelos discentes. Por outro lado, Siddiqui (2013) informa que os alunos concordaram que a maioria dos avaliadores foram corteses e cooperativos durante a prova, tornando-se, assim, um fator positivo para o rendimento nas estações.

Estudos que avaliaram a repercussão do comportamento dos avaliadores durante a realização da prova registraram relato de perturbação entre alguns estudantes, revelando que esse dado não é uma particularidade do curso de Medicina da Universidade de Vassouras. Isso não justifica, contudo, ignorar a adoção de medidas para minimizá-la ou extingui-la, sendo a capacitação pedagógica dos docentes uma das estratégias viáveis.

Acerca de possível estresse ao realizar a prova, 51,5\% dos estudantes informaram que, de fato, se sentiram estressados, valores superiores ao encontrados em estudos de Silva et al. (2019). 
Zimmermann (2019) ratifica que as alterações emocionais dos estudantes ajudaram no autoconhecimento em aprender a lidar com as emoções, contribuindo para seu desenvolvimento pessoal. Já Siddiqui (2013) identificou, entre os motivos para o estresse relacionado ao OSCE, a novidade no formato de uma avaliação diferente daquela realizada em anos anteriores, a falta de instruções a respeito do exame e a nova dinâmica do decorrer da prova. Estudantes sugeriram a necessidade de oportunizar a eles o treinamento de práticas semelhantes às da prova sem, contudo, a atribuição de notas a essas atividades, bem como o fornecimento de orientações prévias sobre o exame (BEVAN; RUSSELL; MARSHALL, 2019).

Bevan, Russell e Marshall (2019) alertam que os exames clínicos objetivamente estruturados são uma experiência estressante para muitos estudantes e profissionais em treinamento. Sugerem, a fim de minimizar esse fato, a realização de um "Pré-OSCE", composto por uma série de estações multifuncionais, com a finalidade de familiarização pelos estudantes com a operacionalização do exame e de domínio de técnicas de controle emocional para as futuras edições do processo avaliativo, quando, de fato, pontuação lhes será conferida.

Ratifica-se aqui o informado por Zimmermann (2019) de que o desafio de ensinar e de avaliar permanece lançado e está intrinsicamente relacionado com a contribuição do olhar do professor e do estudante à construção da espiral do conhecimento na educação.

\section{CONSIDERAÇÕES FINAIS}

Os resultados dessa pesquisa evidenciaram a necessidade de adequações em relação ao tempo destinado às estações do OSCE, ao comportamento dos docentes na avaliação da competência pelo estudante e também à identificação de eventuais iniquidades na realização do método avaliativo.

Conclui-se que há um longo caminho a percorrer pelo curso de Medicina da Universidade de Vassouras na sistematização do OSCE, haja vista a percepção positiva dos estudantes em relação ao exame, ainda que ajustes sejam fundamentais, sinalizando a viabilidade e factibilidade de novas edições.

Há de se destacar a preponderância dos aspectos positivos em relação àqueles identificados com merecedores de ajustes pelos estudantes. Isso sinaliza que as conclusões desse estudo podem subsidiar os gestores acadêmicos do curso de Medicina a analisar criticamente a percepção dos estudantes, a operacionalizar melhorias e a institucionalizar o OSCE, visto, pelos protagonistas do processo ensino-aprendizagem, como método válido na sua avaliação formativa. 


\section{REFERÊNCIAS}

ABBADE, Joélcio Francisco. O Mini-Exercício Clínico (Mini-CEX). In: TIBÉRIO, Iolanda de Fátima Lopes Calvo; DAUD-GALLOTTI, Renta Mahfuz; TRONCON, Luiz Ernesto de Almeida; MARTINS, Milton de Arruda. Avaliação Prática de Habilidades Clínicas em Medicina. São Paulo: Atheneu, 2012. p. 89-96.

ASSOCIAÇÃO MÉDICA MUNDIAL. Declaração de Helsinque. 18aㅗ Assembleia Médica Mundial, Helsinque, Filândia, 1964. Disponível em:

https://www.fcm.unicamp.br/fcm/sites/default/files/declaracao_de_helsinque.pdf. Acesso em: 22 abr. de 2021.

ATARO, Getu; BESSA Solomon; ASAMINEW, Tsedeke. Experience and Challenges of Objective Structured Clinical Examination (OSCE): Perspective of Students and Examiners in a Clinical Department of Ethiopian University. Ethiopian-Journal of Health Science, Jimma, v. 30, n. 3, p. 417-426, 2020.

BEVAN, James.; RUSSELL, Benjamin; MARSHALL, Ben. A new approach to OSCE preparation PrOSCEs. BMC Medical Education, [S.I.], v. 19, n. 126, p. 1-6, 2019. Disponível em: https://bmcmededuc. biomedcentral.com/track/pdf/10.1186/s12909-019-1571-5. Acesso em: 22 abr. 2021. DOI: https://doi.org/10.1186/s12909-019-1571-5.

BRASIL, Ministério da Educação. Conselho Nacional de Saúde. Resolução $n^{\circ} 466$, de 12 de dezembro de 2012. Diário Oficial da União, Brasília, DF. 12 dez. 2012. Disponível em: https://conselho.saude.gov.br/resolucoes/2012/Reso466.pdf. Acesso em 22 abr. 2021.

BRASIL, Ministério da Educação. Resolução no 3, de 20 de junho de 2014. Institui Diretrizes Curriculares Nacionais do Curso de Graduação em Medicina e dá outras providências. Diário Oficial da União, Brasília, DF. 20 jun. 2014. Disponível em:

http://portal.mec.gov.br/cne/arquivos/pdf/Med.pdf. Acesso em: 8 jun. 2020.

BUSTAMANTE, Marco et al. Hacia un nuevo instrumento de evaluación en la carrera de Medicina. Uso del método OSCE. Revista Médica de Chile, Santiago, v. 128, n. 9, p. 10391044, 2000. DOI: http://dx.doi.org/10.4067/S0034-98872000000900013.

CHAVES JUNIOR, Nilson. Relato de experiência com a utilização do OSCE pela disciplina Fundamentos de Cirurgia no curso de Medicina da USS. In: VIII JORNADA SEVERINO SOMBRA. Revista Fluminense de Extensão Universitária, Vassouras, v. 8, n. 2, p. 107, 2018. Disponível em: http://editora.universidadedevassouras.edu.br/index.php/RFEU/issue/view/146. Acesso em: 10 jun. de 2020.

CHAVES, Luís Henrique K. et al. Percepção do estudante sobre a implantação do método OSCE no curso de Odontologia em uma universidade particular. Revista da ABENO, [S.I.], v. 19, n. 2, p. 63-70, 2019. DOI: https://doi.org/10.30979/rev.abeno.v19i2.790. 
EBERHARD, Lydia et al. Analysis of quality and feasibility of an objective stuctured clinical examination. European Journal of Dental Education, [S.I.], v. 15, n. 3, p. 172-8, 2011. DOI: http://dx.doi.org/10.1111/j.1600-0579.2010.00653.x.

FERREIRA, Érica Matos Reis et al. O exame clínico objetivo estruturado no curso de Fisioterapia: o olhar do professor. Revista Docência do Ensino Superior, Belo Horizonte, v. 10, p. 1-8, 2020. DOI: https://doi.org/10.35699/2237-5864.2020.15084.

FRANCO, Camila Ament Giuliani dos Santos et al. OSCE para Competências de Comunicação Clínica e Profissionalismo: Relato de Experiência e Meta-Avaliação. Revista Brasileira de Educação Médica, [S.I.], v. 39, n. 3, p. 433-441, 2015. DOI: https://doi.org/10.1590/1981$52712015 v 39 n 3 e 02832014$.

GONTIJO, Eliane Dias; ALVIM, Cristina Gonçalves; REIS, Zilma Silveira Nogueira. O Desafio da Avaliação na Formação Médica por Competência. Revista Internacional em Língua Portuguesa, [S.I.], n. 33, p. 111-118, 2018. Disponível em: http://www.rilpaulp.org/index.php/rilp/article/view/RILP2018.33.8. Acesso em: 11 jun. 2020. DOI: https://doi.org/10.31492/2184-2043.RILP2018.33/pp.111-118.

GUPTA, Piyush; DEWAN, Pooja; SINGH, Tejinder. Objective Structured Clinical Examination (OSCE) Revisited. Indian Pediatrics, Nova Delhi, v. 47, n. 11, p. 911-20, 2010. Disponível em: https://pubmed.ncbi.nlm.nih.gov/21149898/. Acesso em: 11 jun. 2020. DOI: https://doi.org/10.1007/s13312-010-0155-6.

IDRIS, Saadeldin Ahmed et al. Students' Perception of Surgical Objective Structured Clinical Examination (OSCE) at Final Year MBBS, University of Khartoum, Sudan. Medicine Journal, [S.I.], v. 1, n. 1, p. 17-20, 2014.

KILLINGLEY, Jo; DYSON, Sue. Student midwives' perspectives on efficacy of feedback after objective structured clinical examination. British Journal of Midwifery, [S.I.], v. 24, n. 5, p. 362-

368, 2016. DOI: https://doi.org/10.12968/bjom.2016.24.5.362.

MEGALE, Luiz; GONTIJO, Eliane D.; MOTA, Joaquim Antônio César. Competências Clínicas Essenciais em Pediatria: estão os estudantes aptos a executá-las?. Revista Brasileira de Educação Médica, [S.I.], v. 36, n. 4, p. 478-88, 2012. DOI: https://doi.org/10.1590/S010055022012000600006.

NASIR, Abdulrasheed A. et al. Medical students' perception of objective structured clinical examination: a feedback for process improvement. Journal of Surgical Education, [S.I.], v. 71, n. 5, p. 701-706, 2014. DOI: http://dx.doi.org/10.1016/j.jsurg.2014.02.010.

NEVES, Reinaldo de Souza; BARROS, Ângela Ferreira.; ESPER, Márcia Maria de Araújo; BEZERRA, Thiago José Nunes. Avaliação do exame clínico objetivo estruturado (OSCE) por estudantes e docentes de graduação em enfermagem. Revista Comunicação em Ciências da Saúde, Brasília, v. 27, n. 4, p. 309-316, 2016. DOI: https://doi.org/10.51723/ccs.v27i04.55. 
NULTY, Duncan D. et al. Best practice guidelines for use of OSCEs: maximising value for student learning. Nurse Education Today, v. 1, n. 31, 2011. DOI:

https://doi.org/10.1016/j.nedt.2010.05.006.

PERRENOUD, Phillipe. Avaliação: da excelência à regulação das aprendizagens entre duas lógicas. Porto Alegre: Artmed, 1999.

SAMPAIO, Arabela Maria Barbosa; PRICINOTE, Silvia Cristina Marques Nunes; PEREIRA, Edna Regina Silva. Avaliação clínica estruturada. Revista Eletrônica Gestão \& Saúde, Brasília, v. 5, n. 2, p. 410-426, 2014.

SIDDIQUI, Faisal Ghani. Final year MBBS students' perception for observed structured clinical examination. Journal of College of Physicians and Surgeons Pakistan, Karachi, v. 23, n. 1, p. 20-24, 2013.

SILVA, Dyone Karla Barbosa et al. Percepções de estudantes de medicina sobre o OSCE: análise de seu papel como instrumento de avaliação formativa em uma universidade na Amazônia. Revista Científica Multidisciplinar Núcleo do Conhecimento, São Paulo, v. 2, n. 5, p. 66-85, 2019. Disponível em:

https://www.nucleodoconhecimento.com.br/educacao/percepcoes-de-estudantes. Acesso em: 9 jun. 2020. DOI:

http://doi.org/10.32749/nucleodoconhecimento.com.br/educacao/percepcoes-deestudantes.

SMEE, Sydney. Skill based assessment. British Medical Journal, Londres, v. 326, n. 7.391, p. 703-706, 2003. DOI: http://doi.org/10.1136/bmj.326.7391.703.

SOUZA, Ruy Guilherme Silveira. Atributos Fundamentais dos Procedimentos de Avaliação. In: TIBÉRIO et al. Avaliação Prática de Habilidades Clínicas em Medicina. São Paulo: Atheneu, 2012.

TIBÉRIO, Iolanda de Fátima Lopes Calvo et al. Avaliação Prática de Habilidades Clínicas em Medicina. São Paulo: Atheneu, 2012.

UNIVERSIDADE DE VASSOURAS. Projeto Pedagógico do Curso de Medicina. Pró-Reitoria de Ciências Médicas, Vassouras, RJ. Jan. 2019. Disponível em:

https://www.universidadedevassouras.edu.br/arquivos/graduacao/medicina/20191/PPC_MEDICINA.pdf. Acesso em: 11 jul. 2020.

UNIVERSIDADE DE VASSOURAS. Parecer $\mathrm{n}^{\circ}$.3.908.676, de 10 de março de 2020. Comitê de Ética em Pesquisas, Vassouras, RJ. 10 mar. 2020. Disponível em: https://www.universidadedevassouras.edu.br/pesquisa. Acesso em: 22 abr. 2021.

WILBY, Kyle John; DIAB, Mohammad. Key challenges for implementing a Canadian - based objective structured clinical examination (OSCE) in a Middle Eastern context. Canadian Medical Education Journal, Ottawa, v. 7, n. 3, p. 74-79, 2016. Disponível em: 
https://journalhosting.ucalgary.ca/index.php/cmej/article/view/36720/pdf. Acesso em: 11 jun. 2020.

ZARTMAN, Rosemarie R. et al. Using OSCE-based evaluation: curricular impact over time. Journal of Dental Education, [S.I.], v. 66, n. 12, p. 1323-1330, 2002. DOI: https://onlinelibrary.wiley.com/doi/abs/10.1002/j.0022-0337.2002.66.12.tb03605.x.

ZIMMERMANN, Marlene Harger. Avaliação Clínica Objetiva Estruturada (OSCE) com feedback efetivo e o vídeo feedback: sua interface no ensino e na aprendizagem. 2019. Tese (Doutorado em Ensino de Ciência e Tecnologia) - Universidade Tecnológica Federal do Paraná, Ponta Grossa, 2019. Disponível em: http://repositorio.utfpr.edu.br:8080/jspui/bitstream/1/4169/1/PG_PPGECT_D_Zimmerman n\%2C\%20Marlene\%20Harger_2019.pdf. Acesso em: 22 abr. 2021. 


\section{Nilson Chaves Junior}

Médico pela Universidade Severino Sombra (1977). Mestre em Cirurgia pela Escola Paulista de Medicina (2006). Especialista em Cirurgia pelo Conselho Federal de Medicina (1989). Professor do Curso de Graduação em Medicina da Universidade de Vassouras. Coordenador Adjunto do Curso de Graduação em Medicina da Universidade de Vassouras. Discente do CEDEM/USP.

nchavesjr@me.br

\section{Maria Cristina Almeida de Souza}

Cirurgiã-dentista pela Faculdade de Odontologia de Valença (1992). Doutora em Clínicas pelo C.P.O. São Leopoldo Mandic (2012). Especialista em Saúde Coletiva pelo C.P.O. São Leopoldo Mandic (2007). Docente do curso de graduação em Medicina da Universidade de Vassouras. Docente do mestrado Profissional em Ciências Aplicadas em Saúde da Universidade de Vassouras. Discente do CEDEM/USP.

mcas.souza@uol.com.br

\section{João Carlos de Souza Côrtes Junior}

Médico pela Universidade Federal do Estado do Rio de Janeiro (1997). Doutor (2006) e mestre (2001) em Biologia Celular e Molecular. Pró-Reitor de Ciências Médicas da Universidade de Vassouras. Professor do curso de Medicina da Universidade de Vassouras e professor na Universidade Federal do Estado do Rio de Janeiro (UNIRIO). Discente do CEDEM/USP.

joaocortes@yahoo.com.br

\section{Paula Pitta de Resende Côrtes}

Médica pela Universidade Federal do Estado do Rio de Janeiro (1997). Mestre em Patologia Experimental pela UFF (2004). Coordenadora adjunta do curso de Medicina da Universidade de Vassouras. Professora assistente da Universidade Federal do Estado do Rio de Janeiro (UNIRIO). Discente do CEDEM/USP.

paulapitta@yahoo.com.br

\section{Milton de Arruda Martins}

Médico pela Faculdade de Medicina da USP (1977). Doutor em Patologia pela Faculdade Medicina da USP (1987). Pós-doutorado na Harvard Medical School (1990). Professor Titular de Clínica Médica Geral da Faculdade de Medicina da USP e Diretor do Serviço de Clínica Geral do Hospital das Clínicas da Faculdade de Medicina da USP. Docente do CEDEM/USP. mmartins@usp.br 